$B_{4}{ }^{q} \sim \int\left(\varrho_{\mathrm{e}} / r_{\mathrm{e}}{ }^{3}\right) Y_{2}^{q} \mathrm{~d} \tau_{\mathrm{e}} ; \quad B_{16}{ }^{q} \sim \int\left(\varrho_{\mathrm{e}} / r_{\mathrm{e}}{ }^{5}\right) Y_{4}^{q} \mathrm{~d} \tau_{\mathrm{e}}$,

wobei $\varrho_{\mathrm{e}}$ die Ladungsdichte der den Kern umgebenden Elektronen und $Y_{n}^{q}$ die Kugelflächenfunktionen sind.

Nach ${ }^{2}$ und ${ }^{3}$ erhält $1 / \tau_{m}$ die Form

$\frac{1}{\tau_{m}} \sim \sum_{q}(\langle m|F| m \pm q\rangle)^{2}=\sum_{q} C_{a}(I, m, m \pm q) \cdot B_{a^{q}}$.

Die Form und Breite der Protonenlinien wird dann wesentlich durch die Faktoren $C(I, m, m \pm q)$ bestimmt. Für das $\mathrm{GeH}_{4}$ ergeben sich daraus die Folgerungen:

1. Im Falle reiner Tetraedersymmetrie des elektrischen Feldes verschwinden alle Gradienten $B_{4}{ }^{q}$ aus Symmetriegründen. Eine Quadrupolverbreiterung erfordert also eine Zerstörung der Symmetrie, was durch Stöße mit der Umgebung geschehen könnte. Bei einer vollständigen Zerstörung der Symmetrie sind alle Übergänge $\Delta m_{\mathrm{Ge}}= \pm 1, \pm 2$ möglich. Die Koeffizienten $C$ verhalten sich dann wie $720: 1488: 1460: 1368: 1200$ für $m_{\mathrm{Ge}}=9 / 2$ bis $m_{\mathrm{Ge}}=1 / 2$, und man erhält die Linienformen der Abb. 2 a, die mit den beobachteten nicht übereinstimmen.

2. Falls das fluktuierende elektrische Feld am Ort des $\mathrm{Ge}$ eine $\mathrm{C}_{3 \mathrm{v}^{-}}$oder $\mathrm{D}_{2 \mathrm{~h}}$-Symmetrie hat, zeigt die Gruppentheorie, daß nur die Komponenten $B_{4}{ }^{ \pm 2}$ nicht verschwinden. Die zugeordnete Quadrupolkomponente führt nur zu Übergängen $\Delta m= \pm 2$. Es verhalten sich die $C(I, m, m \pm 2)$ wie $144: 336: 648: 936: 1104$,

4 A. Abragam, The Principles of Nuclear Magnetism, Clarendon Press, Oxford 1960. und man erhält die Linienbreiten der Abb. 2 b, die mit der Beobachtung übereinstimmen.

3. Eine Hexadecapolwechselwirkung würde auch im Falle der Tetraedersymmetrie Übergänge $\Delta m= \pm 4$ geben. Die $C(I, m, m \pm 4)$ führen dann zu den Linienbreiten der Abb. $2 \mathrm{c}$, die mit den experimentellen übereinstimmen. Allerdings zeigt eine Abschätzung, daß die Hexadecapolwechselwirkung um den Faktor $10^{-8}$ kleiner ist als die Quadrupolwechselwirkung und zu Relaxationszeiten von $T_{2} \sim 10^{12} \mathrm{~s}$ führen würde. Bei den Abb. 2 a, 2 b, 2 c wurde die zusätzliche Verbreiterung $1 / T^{\text {inh }}$ infolge der Magnetfeldinhomogenität berücksichtigt, und zwar unter der Annahme, daß diese ebenfalls auf eine Lorentz-Verteilung führt. Nach Abragam ${ }^{4}$ ist dann die beobachtete Halbwertsbreite gegeben durch:

$$
1 / T_{2}=1 / T_{2}{ }^{\prime}+1 / T_{2}{ }^{i n h}+1 / \tau_{m} .
$$

Es wurde angenommen $1 / T_{2}{ }^{\prime} \approx 0,1 / T_{2}{ }^{\mathrm{inh}} \approx 0,5 \mathrm{~Hz}$. Im Gegensatz zum $\mathrm{GeH}_{4}$ wurde von Packer und Muetterties ${ }^{5}$ bei $\mathrm{NbF}_{6}{ }^{-}\left(I_{\mathrm{Nb}^{33}}{ }^{93}=9 / 2\right)$ der Abb. 2 a gehorchende Linienbreiten beobachtet, die mit der Annahme totaler Symmetriezerstörung ${ }^{2,3}$ erklärt werden können.

Herrn Prof. Dr. Тн. Förster möchten wir für die Förderung dieser Arbeit und der Deutschen Forschungsgemeinschaft für finanzielle Unterstützung herzlich danken.

5 K. J. Packer u. E. L. Muetterties, J. Am. Chem. Soc. 85, 3035 [1963].

\section{Zur Protonenrelaxation des Benzols}

\author{
K.-H. WeISS
}

Physikalisches Institut der Universität Leipzig

(Z. Naturforschg. 21 a, 853-854 [1966] ; eingegangen am 2. Mai 1966)

Nach Veröffentlichung der Arbeit ${ }^{1}$ im Jahre 1964 sind von mehreren Autoren longitudinale Relaxationszeiten $T_{1}$ von Benzol gemessen worden, welche sämtlich die bis dahin in der Literatur vorliegenden Ergebnisse bestätigen. Als Beispiel seien die Resultate von HaEBERlen, Hausser, Noack und Maier ${ }^{2}$ genannt, die im Frequenzintervall zwischen $50 \mathrm{kHz}$ und $100 \mathrm{MHz}$ bei $25^{\circ} \mathrm{C}$ $T_{1}$-Werte von etwa $19,5 \mathrm{~s}$ fanden. Im Gegensatz dazu wurden in dem genannten Zeitraum keine weiteren transversalen Relaxationszeiten $T_{2}$ von Benzol publiziert.

Da der von Meiboom und $\mathrm{Gill}^{3}$, Cutler ${ }^{4}$, von Haupt und Müller-W $\mathrm{W}_{\mathrm{ARMUTH}}{ }^{5}$ und von uns gleichermaßen ge-

1 K.-H. WeIss, Z. Naturforschg. 19 a, 1424 [1964].

2 U. Haeberlen, R. Hausser, F. Noack u. G. Maier, Phys. Letters 12, 306 [1964].

3 S. Мегвоом u. D. Gill, Rev. Sci. Instr. 29, 688 [1958].

4 D. Cutler, Thesis, London (1960). fundene Unterschied zwischen $T_{1}$ und $T_{2}$ bei Benzol weder durch eine Wechselwirkung innerhalb des Benzols (insbesondere mit dem natürlichen Deuteronengehalt) - noch durch Wandeinflüsse erklärt werden konnte, haben wir alle in Frage kommenden apparativen Bedingungen systematisch variiert und dabei die im folgenden angeführten Ergebnisse gefunden.

Die Untersuchungen wurden mit dem gleichen Spektrometer wie in ${ }^{1}$ durchgeführt. Der verwendete Elektromagnet besaß eine Stabilität von mindestens $10^{-5}$ pro Stunde. Durch Veränderung der $\pi$-Impulsfrequenz $N$ innerhalb der Impulsgruppe nach C CarR und Purcell ${ }^{6}$ (modifiziert nach Меiвoom und $\mathrm{Gill}^{3}$ ) und durch die Wahl unterschiedlicher $H_{0}$-Feldgradienten $G$ haben wir zunächst den Selbstdiffusionseinfluß abgeschätzt und für unsere Meßbedingungen $(G=0,5 \mathrm{Gau} / \mathrm{cm}$, $N=500 \mathrm{~Hz}$ ) mit Sicherheit ausgeschlossen. Um einem von Hausser und NoAcK ${ }^{7}$ vermuteten Strahlungsdämpfungseinfluß ganz sicher zu begegnen, wurden die bereits in ${ }^{1}$ erwähnten Experimente wesentlich erweitert.

5 J. Haupt u. W. Müller-W 405 [1962].

6 H. Y. Carr u. R. M. Purcell, Phys. Rev. 94, 630 [1954].

7 R. Hausser u. F. Noack, Z. Naturforschg. 19 a, 1521 [1964]. 
Durch Entdämpfen des die Probe enthaltenden Schwingungskreises (Einspulenanordnung) war es möglich, bei Güten zwischen 40 und 1300 zu messen. In dem gesamten Intervall ergaben sich jedoch immer die gleichen $T_{2}$-Werte, was den Einfluß einer Strahlungsdämpfung sicher ausschließt. Bei Verstimmung der $H_{0}$-Feldstärke bzw. der Frequenz der am Eingang des Spektrometers angelegten 16-MHz-Spannung (Mutteroszillator) um $\pm 6 \%$ änderten sich die $T_{2}$-Werte um weniger als $3 \%$. Gegenüber Fehljustierungen der $\pi$-Impulsbreiten bzw. des Abstandes zwischen dem $\pi / 2$-Impuls und den nachfolgenden $\pi$-Impulsen der CARR-Gruppe um $\pm 10 \%$ erwiesen sich die $T_{2}$-Messungen als gänzlich unempfindlich.

Einen starken Einfluß zeigten hingegen geringfügige periodische Phasenmodulationen des Mutteroszillators. Und zwar genügten bei sinusförmiger Modulation mit Modulationsfrequenzen zwischen $0,1 \mathrm{~Hz}$ und etwa $10 \mathrm{kHz}$ Phasenhübe der Größenordnung $10^{-3}$ bis $10^{-4}$, um die ursprünglich exponentiellen Echoserien stark nichtmonoton und sehr rasch abklingen zu lassen. Solche Phasenmodulationen ließen sich aber im Ausgang der als Mutteroszillator verwendeten Normalfrequenzanlage CFQ BN 775 D nachweisen. In Übereinstimmung damit ergab sich eine $T_{2}$-Verlängerung bei Übergang zu einem Sender, der diese Modulationen nicht besitzt. Als unabhängig von der Wahl des Mutteroszillators erwies sich jedoch die relativ große Streuung der Einzelwerte, die sich stets auf die gleiche Weise unsymmetrisch um ihren häufigsten Wert gruppierten und nicht etwa eine $\mathrm{G}_{\text {Auss-Verteilung bildeten, wie man }}$ es bei rein zufälligen Abweichungen erwarten sollte. Diese Verteilung mußte demnach wesentlich apparativ bedingt sein und ließ sich schließlich durch die Annahme statistischer Schwankungen der $\pi$-Impulsbreiten und -abstände der Größenordnung $10^{-3}$, wie sie bei Impulsgeneratoren mit analogen Schaltungen zu erwarten sind ${ }^{8}$, quantitativ erklären ${ }^{9}$. Es zeigte sich, daß die

8 H. SCHÄFER, Intern. Elektron. Rundschau 9, 485 [1965].

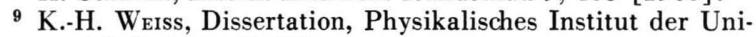
versität Leipzig (1966).

10 H. Sprinz, Dissertation, Physikalisches Institut der Universität Leipzig (1966).

10a Nach einer privaten Mitteilung vom 18.2.1966 fanden Kosfeld und Oehrmann (Aachen) ebenfalls die Gleichheit beider Relaxationszeiten $T_{1}$ und $T_{2}$ in Benzol, und zwar statistischen Schwankungen der genannten Impulsparameter immer Verkürzungen der gemessenen $T_{2}$-Werte zur Folge haben und daß bei einer Standardabweichung von $1,7 \cdot 10^{-3}$ und einem wahren $T_{2}=20 \mathrm{~s}$ etwa nur $20 \%$ aller Einzelmessungen in das Intervall zwischen $T_{2}$ und $0,9 \cdot T_{2}$ fallen. Für eine Standardabweichung von $1 \cdot 10^{-3}$ und verschiedene $T_{2}$ sind die entsprechenden Bruchteile in Tab. 1 zusammengestellt.

\begin{tabular}{|c|c|}
\hline$T_{2}[\mathrm{~s}]$ & $p$ \\
\hline 3 & $99 \%$ \\
10 & $96 \%$ \\
20 & $71 \%$ \\
40 & $35 \%$ \\
60 & $20 \%$ \\
80 & $12 \%$ \\
100 & $9 \%$ \\
\hline
\end{tabular}

Tab. 1. Bruchteil $p$ der in das Intervall $T_{2} \ldots 0,9 \cdot T_{2}$ fallenden Einzelmessungen bei einer relativen Standardabweichung der $\pi$-Impulsbreiten und -abstände von $1 \cdot 10^{-3}$ für verschiedene $T_{2}$.

Insbesondere folgt hieraus, daß aus einer Vielzahl von Einzelmessungen die längsten $T_{2}$-Werte viel mehr dem wahren $T_{2}$ entsprechen als die am häufigsten gemessenen. Auf diese Weise gelang es uns, bei $23{ }^{\circ} \mathrm{C}$ und $16 \mathrm{MHz}$ die transversale Relaxationszeit des Benzols zu $T_{2}=(18,7 \pm 0,3) \mathrm{s}$ zu bestimmen und damit ihre Übereinstimmung mit der longitudinalen Relaxationszeit $T_{1}=(19,5 \pm 1) \mathrm{s}$ nachzuweisen. Entsprechende Messungen führten wir an dem von SPRINZ ${ }^{10}$ beschriebenen Spin-Echo-Spektrometer bei $40 \mathrm{kHz}$ aus und fanden auch hier die Gleichheit beider Relaxationszeiten (zwischen $20{ }^{\circ} \mathrm{C}$ und $90{ }^{\circ} \mathrm{C}$ ) ${ }^{10 a}$. Kontrollmessungen von $T_{1}$ und $T_{2}$ an Wasser zwischen $20^{\circ} \mathrm{C}$ und $200^{\circ} \mathrm{C}$ ergaben Úbereinstimmung mit den bereits früher veröffentlichten Werten ${ }^{11,12}$.

Herrn Prof. Dr. H. Pfeifer möchte ich für seine stete Unterstützung und für die vielen Hinweise während des gesamten Verlaufes dieser Arbeit herzlich danken. Danken möchte ich auch allen meinen Kollegen, mit denen mich in den vergangenen Jahren eine angenehme Zusammenarbeit verband.

bei $60 \mathrm{MHz}$ und in dem Temperaturintervall zwischen $-20^{\circ} \mathrm{C}$ und $140^{\circ} \mathrm{C}$. - Vgl. R. Kosfeld u. L. Oehrmann, Z. Naturforschg. 21 a, 855 [1966].

11 H. Pfeifer, in Hochfrequenzspektroskopie, Akademie-Verlag, Berlin 1961, S. $58 \mathrm{ff}$.

12 R. Hausser, Dissertation, Technische Hochschule Stuttgart (1964). 